\title{
MIXED PROBLEM WITH NONLOCAL BOUNDARY CONDITIONS FOR A THIRD-ORDER PARTIAL DIFFERENTIAL EQUATION OF MIXED TYPE
}

\author{
M. DENCHE and A. L. MARHOUNE
}

(Received 7 January 2000)

\begin{abstract}
We study a mixed problem with integral boundary conditions for a third-order partial differential equation of mixed type. We prove the existence and uniqueness of the solution. The proof is based on two-sided a priori estimates and on the density of the range of the operator generated by the considered problem.
\end{abstract}

2000 Mathematics Subject Classification. 35B45, 35K20, 35M10.

1. Introduction. In the rectangle $\Omega=(0, \ell) \times(0, T)$, we consider the equation

$$
\mathscr{L} u=\frac{\partial^{2} u}{\partial t^{2}}-\frac{\partial}{\partial x}\left(a(x, t) \frac{\partial^{2} u}{\partial x \partial t}\right)=f(x, t),
$$

where $a(x, t)$ is bounded with $0<a_{0}<a(x, t) \leq a_{1}$ and has bounded partial derivatives such that $0<a_{2} \leq \partial a(x, t) / \partial t \leq a_{3}$ and $0<a_{4} \leq \partial a(x, t) / \partial x \leq a_{5}$ for $(x, t) \in \bar{\Omega}$.

To (1.1) we add the initial conditions

$$
l_{1} u=u(x, 0)=\varphi(x), \quad l_{2} u=\frac{\partial u}{\partial t}(x, 0)=\psi(x), \quad x \in(0, \ell)
$$

the Dirichlet condition

$$
u(0, t)=0, \quad t \in(0, T)
$$

and the integral condition

$$
\int_{0}^{\ell} u(\xi, t) d \xi=0, \quad t \in(0, T),
$$

where $\varphi$ and $\psi$ are known functions which satisfy the compatibility conditions given by (1.3) and (1.4), that is,

$$
\varphi(0)=0, \quad \int_{0}^{\ell} \varphi(x) d x=0, \quad \psi(0)=0, \quad \int_{0}^{\ell} \psi(x) d x=0 .
$$

Boundary-value problems for parabolic equations with integral boundary conditions are investigated by Batten [1], Bouziani and Benouar [2], Cannon [3, 4], Perez Esteva and van der Hoeck [5], Ionkin [8], Kamynin [9], Kartynnik [10], Shi [11], Yurchuk [13], and many references therein. We remark that integral boundary conditions for evolution problems have various applications in chemical engineering, thermoelasticity, underground water flow and population dynamics; see for example, [6, 7, 11, 12]. 
The present paper is devoted to the study of a mixed problem with boundary integral conditions for a third-order partial differential equation of mixed type.

We associate to problem (1.1), (1.2), (1.3), and (1.4) the operator $L=\left(\mathscr{L}, l_{1}, l_{2}\right)$, defined from $E$ into $F$, where $E$ is the Banach space of functions $u \in L_{2}(\Omega)$, satisfying (1.3) and (1.4), with the finite norm

$$
\begin{aligned}
\|u\|_{E}^{2}= & \int_{\Omega}(\ell-x)^{2}\left[\left|\frac{\partial^{2} u}{\partial t^{2}}\right|^{2}+\left|\frac{\partial^{3} u}{\partial x^{2} \partial t}\right|^{2}\right] d x d t \\
& +\sup _{0 \leq t \leq T} \int_{0}^{\ell}(\ell-x)^{2}\left[\left|\frac{\partial^{2} u}{\partial x \partial t}\right|^{2}+\left|\frac{\partial u}{\partial x}\right|^{2}\right] d x+\sup _{0 \leq t \leq T} \int_{0}^{\ell}\left[\left|\frac{\partial u}{\partial t}\right|^{2}+|u|^{2}\right] d x,
\end{aligned}
$$

and $F$ is the Hilbert space of vector-valued functions $\mathscr{F}=(f, \varphi, \psi)$ obtained by completion of the space $L_{2}(\Omega) \times W_{2}^{2}(0, \ell) \times W_{2}^{2}(0, \ell)$ with respect to the norm

$$
\begin{aligned}
\|\mathscr{F}\|_{F}^{2} & =\|(f, \varphi, \psi)\|_{F}^{2} \\
& =\int_{\Omega}(\ell-x)^{2}|f|^{2} d x d t+\int_{0}^{\ell}(\ell-x)^{2}\left[\left|\frac{d \varphi}{d x}\right|^{2}+\left|\frac{d \psi}{d x}\right|^{2}\right] d x+\int_{0}^{\ell}\left[|\varphi|^{2}+|\psi|^{2}\right] d x .
\end{aligned}
$$

Using the energy inequalities method proposed in [13], we establish two-sided a priori estimates. Then, we prove that the operator $L$ is a linear homeomorphism between the spaces $E$ and $F$.

\section{Two-sided a priori estimates}

THEOREM 2.1. For any function $u \in E$, there is the a priori estimate

$$
\|L u\|_{F} \leq c\|u\|_{E},
$$

where the constant $c$ is independent of $u$.

Proof. Using (1.1) and the initial conditions (1.2), we obtain

$$
\begin{gathered}
\int_{\Omega}(\ell-x)^{2}|\mathscr{L u}|^{2} d x d t \leq 3 \int_{\Omega}(\ell-x)^{2}\left[\left|\frac{\partial^{2} u}{\partial t^{2}}\right|^{2}+a_{5}^{2}\left|\frac{\partial^{2} u}{\partial x \partial t}\right|^{2}+a_{1}^{2}\left|\frac{\partial^{3} u}{\partial x^{2} \partial t}\right|^{2}\right] d x d t, \\
\int_{0}^{\ell}(\ell-x)^{2}\left[\left|\frac{d \psi}{d x}\right|^{2}+\left|\frac{d \varphi}{d x}\right|^{2}\right] d x \leq \sup _{0 \leq t \leq T} \int_{0}^{\ell}(\ell-x)^{2}\left[\left|\frac{\partial^{2} u}{\partial x \partial t}\right|^{2}+\left|\frac{\partial u}{\partial x}\right|^{2}\right] d x, \\
\int_{0}^{\ell}\left[|\psi|^{2}+|\varphi|^{2}\right] d x \leq \sup _{0 \leq t \leq T} \int_{0}^{\ell}\left[\left|\frac{\partial u}{\partial t}\right|^{2}+|u|^{2}\right] d x .
\end{gathered}
$$

Combining the inequalities (2.2), we obtain (2.1) for $u \in E$.

THEOREM 2.2. For any function $u \in E$, there is the a priori estimate

$$
\|u\|_{E} \leq \alpha\|L u\|_{F}
$$

with the constant

$$
\alpha=\frac{\max \left(167 / 10, a_{1}\right)}{\min \left(\exp (-c T) / 20, \exp (-c T) a_{0}^{2} / 15\right)},
$$


and $c$ is such that

$$
c \geq 1, \quad c a_{0}-1 \geq a_{3}+2 a_{5}^{2} .
$$

Before proving this theorem, we first give the following two lemmas.

LEMMA 2.3. For $u \in E$ satisfying the first condition in (1.2),

$$
\begin{aligned}
\frac{1}{2} \int_{0}^{\tau} \int_{0}^{\ell}(\ell-x)^{2} \exp (-c t)\left|\frac{\partial^{2} u}{\partial x \partial t}\right|^{2} d x d t+\frac{c-1}{2} \int_{0}^{\tau} \int_{0}^{\ell}(\ell-x)^{2} \exp (-c t)\left|\frac{\partial u}{\partial x}\right|^{2} d x d t \\
\geq \frac{1}{2} \int_{0}^{\ell}(\ell-x)^{2} \exp (-c \tau)\left|\frac{\partial u}{\partial x}(x, \tau)\right|^{2} d x-\frac{1}{2} \int_{0}^{\ell}(\ell-x)^{2}\left|\frac{d \varphi}{d x}\right|^{2} d x
\end{aligned}
$$

Proof. Starting from

$$
\int_{0}^{\tau} \int_{0}^{\ell}(\ell-x)^{2} \exp (-c t) \frac{\partial}{\partial t}\left(\frac{\partial u}{\partial x}\right) \frac{\partial \bar{u}}{\partial x} d x d t
$$

then integrating by parts and using elementary inequalities, we obtain (2.6).

LEMMA 2.4. For $u \in E$ satisfying the initial conditions (1.2),

$$
\int_{0}^{\ell} \exp (-c \tau)|u(x, \tau)|^{2} d x \leq \int_{0}^{\tau} \int_{0}^{\ell} \exp (-c t)\left|\frac{\partial u}{\partial t}\right|^{2} d x d t+\int_{0}^{\ell}|\varphi|^{2} d x,
$$

with $c \geq 1$.

Proof. Integrating by parts the expression

$$
\int_{0}^{\tau} \int_{0}^{\ell} \exp (-c t) u \frac{\partial \bar{u}}{\partial t} d x d t
$$

and using elementary inequalities yield (2.8).

REMARK 2.5. We note that Lemmas 2.3 and 2.4 hold for weaker conditions on $u$.

Proof of Theorem 2.2. First, define

$$
D(L)=\left\{u \in E \mid \frac{\partial^{5} u}{\partial x^{2} \partial t^{3}} \in L_{2}(\Omega)\right\}, \quad M u=(\ell-x)^{2} \frac{\partial^{2} u}{\partial t^{2}}+2(\ell-x) J \frac{\partial^{2} u}{\partial t^{2}},
$$

where

$$
J u=\int_{0}^{x} u(\xi, t) d \xi .
$$

We consider for $u \in D(L)$ the quadratic formula

$$
\operatorname{Re} \int_{0}^{\tau} \int_{0}^{\ell} \exp (-c t) \mathscr{L} u \overline{M u} d x d t
$$

with the constant $c$ satisfying (2.5), obtained by multiplying (1.1) by $\exp (-c t) M u$, by 
integrating over $\Omega^{\tau}$, where $\Omega^{T}=(0, \ell) \times(0, \tau)$, with $0 \leq \tau \leq T$, and by taking the real part. Integrating by parts (2.12) with the use of boundary conditions (1.3) and (1.4), we obtain

$$
\begin{aligned}
\operatorname{Re} \int_{0}^{T} \int_{0}^{\ell} \exp (-c t) \mathscr{L} u M \bar{u} d x d t \\
=\int_{0}^{\tau} \int_{0}^{\ell}(\ell-x)^{2} \exp (-c t)\left|\frac{\partial^{2} u}{\partial t^{2}}\right|^{2} d x d t+\frac{1}{2} \int_{0}^{\tau} \int_{0}^{\ell} \exp (-c t)\left|J \frac{\partial^{2} u}{\partial t^{2}}\right|^{2} d x d t \\
\quad+\operatorname{Re} \int_{0}^{\tau} \int_{0}^{\ell}(\ell-x)^{2} \exp (-c t) a \frac{\partial^{2} u}{\partial x \partial t} \frac{\partial}{\partial t}\left(\frac{\partial^{2} \bar{u}}{\partial x \partial t}\right) d x d t \\
\quad+2 \operatorname{Re} \int_{0}^{\tau} \int_{0}^{\ell} \exp (-c t) \frac{\partial u}{\partial t} a \frac{\partial^{2} \bar{u}}{\partial t^{2}} d x d t \\
\quad+2 \operatorname{Re} \int_{0}^{\tau} \int_{0}^{\ell} \exp (-c t) \frac{\partial a}{\partial x} \frac{\partial u}{\partial t} J \frac{\partial^{2} \bar{u}}{\partial t^{2}} d x d t .
\end{aligned}
$$

On the other hand, by using the elementary inequalities we get

$$
\begin{aligned}
\operatorname{Re} \int_{0}^{T} \int_{0}^{\ell} & \exp (-c t) \mathscr{L} u M \bar{u} d x d t \\
\geq & \int_{0}^{\tau} \int_{0}^{\ell}(\ell-x)^{2} \exp (-c t)\left|\frac{\partial^{2} u}{\partial t^{2}}\right|^{2} d x d t \\
& +\operatorname{Re} \int_{0}^{\tau} \int_{0}^{\ell}(\ell-x)^{2} \exp (-c t) a \frac{\partial^{2} u}{\partial x \partial t} \frac{\partial}{\partial t}\left(\frac{\partial^{2} \bar{u}}{\partial x \partial t}\right) d x d t \\
& +2 \operatorname{Re} \int_{0}^{\tau} \int_{0}^{\ell} \exp (-c t) \frac{\partial u}{\partial t} a^{\frac{\partial^{2}}{u}} \frac{\partial t^{2}}{d x} d t \\
& -2 \operatorname{Re} \int_{0}^{\tau} \int_{0}^{\ell} \exp (-c t)\left|\frac{\partial a}{\partial x}\right|^{2}\left|\frac{\partial u}{\partial t}\right|^{2} d x d t .
\end{aligned}
$$

Again, integrating by parts the second and third terms of the right-hand side of the inequality (2.14) and taking into account the initial conditions (1.2) give

$$
\begin{aligned}
\operatorname{Re} & \int_{0}^{\tau} \int_{0}^{\ell} \exp (-c t) \mathscr{L} u M \bar{u} d x d t+\int_{0}^{\ell} a(x, 0)|\psi|^{2} d x+\frac{1}{2} \int_{0}^{\ell} a(x, 0)(\ell-x)^{2}\left|\frac{d \psi}{d x}\right|^{2} d x d t \\
\geq & \int_{0}^{\tau} \int_{0}^{\ell} \exp (-c t)(\ell-x)^{2}\left|\frac{\partial^{2} u}{\partial t^{2}}\right|^{2} d x d t-2 \int_{0}^{\tau} \int_{0}^{\ell} \exp (-c t)\left|\frac{\partial a}{\partial x}\right|^{2}\left|\frac{\partial u}{\partial t}\right|^{2} d x d t \\
& +\frac{1}{2} \int_{0}^{\ell} a(x, \tau) \exp (-c \tau)(\ell-x)\left|\frac{\partial^{2} u}{\partial x \partial t}(x, \tau)\right|^{2} d x \\
& -\frac{1}{2} \int_{0}^{\tau} \int_{0}^{\ell} \exp (-c t) \frac{\partial a}{\partial t}(\ell-x)^{2}\left|\frac{\partial^{2} u}{\partial x \partial t}\right|^{2} d x d t \\
& +\frac{c}{2} \int_{0}^{\tau} \int_{0}^{\ell} \exp (-c t)(\ell-x)^{2} a\left|\frac{\partial^{2} u}{\partial x \partial t}\right|^{2} d x d t+\int_{0}^{\ell} \exp (-c \tau) a(x, \tau)\left|\frac{\partial u}{\partial t}(x, \tau)\right|^{2} d x \\
& -\int_{0}^{\tau} \int_{0}^{\ell} \exp (-c t) \frac{\partial a}{\partial t}\left|\frac{\partial u}{\partial t}\right|^{2} d x d t+c \int_{0}^{\tau} \int_{0}^{\ell} \exp (-c t) a\left|\frac{\partial u}{\partial t}\right|^{2} d x d t .
\end{aligned}
$$


By using the elementary inequalities on the first integral in the left-hand side of (2.15), we obtain

$$
\begin{aligned}
& \frac{33}{2} \int_{0}^{\tau} \int_{0}^{\ell} \exp (-c t)(\ell-x)^{2}|\mathscr{L} u|^{2} d x d t+\frac{3}{4} \int_{0}^{\tau} \int_{0}^{\ell} \exp (-c t)(\ell-x)^{2}\left|\frac{\partial^{2} u}{\partial t^{2}}\right|^{2} d x d t \\
& \quad+\int_{0}^{\ell} a(x, 0)|\psi|^{2} d x+\frac{1}{2} \int_{0}^{\ell} a(x, 0)(\ell-x)^{2}\left|\frac{d \psi}{d x}\right|^{2} d x \\
& \geq \int_{0}^{\tau} \int_{0}^{\ell} \exp (-c t)(\ell-x)^{2}\left|\frac{\partial^{2} u}{\partial t^{2}}\right|^{2} d x d t-2 \int_{0}^{\tau} \int_{0}^{\ell} \exp (-c t)\left|\frac{\partial a}{\partial x}\right|^{2}\left|\frac{\partial u}{\partial t}\right|^{2} d x d t \\
& \quad+\frac{1}{2} \int_{0}^{\ell} \exp (-c \tau)(\ell-x)^{2}\left|\frac{\partial^{2} u(x, \tau)}{\partial x \partial t}\right|^{2} d x-\frac{1}{2} \int_{0}^{\tau} \int_{0}^{\ell} \exp (-c t)(\ell-x)^{2} \frac{\partial a}{\partial t}\left|\frac{\partial^{2} u}{\partial x \partial t}\right|^{2} d x d t \\
& \quad+\frac{c}{2} \int_{0}^{\tau} \int_{0}^{\ell} \exp (-c t)(\ell-x)^{2} a\left|\frac{\partial^{2} u}{\partial x \partial t}\right|^{2} d x d t+\int_{0}^{\ell} \exp (-c \tau) a(x, \tau)\left|\frac{\partial u(x, \tau)}{\partial t}\right|^{2} d x \\
& \quad-\int_{0}^{\tau} \int_{0}^{\ell} \exp (-c t) \frac{\partial a}{\partial t}\left|\frac{\partial u}{\partial t}\right|^{2} d x d t+c \int_{0}^{\tau} \int_{0}^{\ell} \exp (-c t) a\left|\frac{\partial u}{\partial t}\right|^{2} d x d t .
\end{aligned}
$$

Now, from (1.1) we have

$$
\begin{aligned}
\frac{1}{5} \int_{0}^{\tau} \int_{0}^{\ell} \exp (-c t)(\ell-x)^{2}|\mathscr{L} u|^{2} d x d t \\
\quad+\frac{1}{5} \int_{0}^{\tau} \int_{0}^{\ell} \exp (-c t)(\ell-x)^{2}\left|\frac{\partial a}{\partial x}\right|^{2}\left|\frac{\partial^{2} u}{\partial x \partial t}\right|^{2} d x d t \\
\quad+\frac{1}{5} \int_{0}^{\tau} \int_{0}^{\ell} \exp (-c t)(\ell-x)^{2}\left|\frac{\partial^{2} u}{\partial t^{2}}\right|^{2} d x d t \\
\geq \frac{1}{15} \int_{0}^{\tau} \int_{0}^{\ell} \exp (-c t)(\ell-x)^{2} a^{2}\left|\frac{\partial^{3} u}{\partial x^{2} \partial t}\right|^{2} d x d t
\end{aligned}
$$

Combining inequalities (2.16), (2.17), and Lemmas 2.3 and 2.4, we get

$$
\begin{aligned}
& \frac{167}{10} \int_{\Omega}(\ell-x)^{2}|\mathscr{L} u|^{2} d x d t+\frac{a_{1}}{2} \int_{0}^{\ell}(\ell-x)^{2}\left|\frac{d \psi}{d x}\right|^{2} d x \\
& +a_{1} \int_{0}^{\ell}|\psi|^{2} d x+\frac{1}{2} \int_{0}^{\ell}(\ell-x)^{2}\left|\frac{d \varphi}{d x}\right|^{2} d x+\int_{0}^{\ell}|\varphi|^{2} d x \\
& \geq \exp (-c T)\left(\frac{1}{20} \int_{0}^{\tau} \int_{0}^{\ell}(\ell-x)^{2}\left|\frac{\partial^{2} u}{\partial t^{2}}\right|^{2} d x d t+\frac{1}{2} \int_{0}^{\ell}(\ell-x)^{2}\left|\frac{\partial^{2} u}{\partial x \partial t}(x, \tau)\right|^{2} d x d t\right. \\
& +\int_{0}^{\ell}|u(x, \tau)|^{2} d x+a_{0} \int_{0}^{\ell}\left|\frac{\partial u}{\partial t}(x, \tau)\right|^{2} d x+\frac{1}{2} \int_{0}^{\ell}(\ell-x)^{2}\left|\frac{\partial u}{\partial x}(x, \tau)\right|^{2} d x \\
& \left.+\frac{a_{0}^{2}}{15} \int_{0}^{\tau} \int_{0}^{\ell}(\ell-x)^{2}\left|\frac{\partial^{3} u}{\partial x^{2} \partial t}\right|^{2} d x d t\right) .
\end{aligned}
$$

As the left-hand side of (2.18) is independent of $\tau$, by replacing the right-hand side by its upper bound with respect to $\tau$ in the interval $[0, T]$, we obtain the desired inequality. 
3. Solvability of the problem. From estimates (2.1) and (2.3) it follows that the operator $L: E \rightarrow F$ is continuous and its range is closed in $F$. Therefore, the inverse operator $L^{-1}$ exists and is continuous from the closed subspace $R(L)$ onto $E$, which means that $L$ is a homomorphism from $E$ onto $R(L)$. To obtain the uniqueness of solution, it remains to show that $R(L)=F$. The proof is based on the following lemma.

LEMMA 3.1. Suppose that $\partial^{3} a / \partial x^{2} \partial t$ is also bounded. Let $D_{0}(L)=\left\{u \in D(L): l_{1} u=0\right.$, $\left.l_{2} u=0\right\}$. If for $u \in D_{0}(L)$ and some $\omega \in L_{2}(\Omega)$,

$$
\int_{\Omega}(\ell-x) \mathscr{L} u \varpi d x d t=0
$$

then $\omega=0$.

Proof. From (3.1) we have

$$
\int_{\Omega}(\ell-x) \frac{\partial^{2} u}{\partial t^{2}} \varpi d x d t=\int_{\Omega}(\ell-x) \frac{\partial}{\partial x}\left(a \frac{\partial^{2} u}{\partial x \partial t}\right) \varpi d x d t .
$$

If we introduce the smoothing operators with respect to $t$ (see [13]) $J_{\xi}^{-1}=(I+\xi(\partial / \partial t))^{-1}$ and $\left(J_{\xi}^{-1}\right)^{*}$, then these operators provide the solutions of the respective problems

$$
\begin{gathered}
\xi \frac{d g_{\xi}(t)}{d t}+g_{\xi}(t)=g(t),\left.\quad g_{\xi}(t)\right|_{t=0}=0, \\
-\xi \frac{d g_{\xi}^{*}(t)}{d t}+g_{\xi}^{*}(t)=g(t),\left.\quad g_{\xi}^{*}(t)\right|_{t=T}=0,
\end{gathered}
$$

and also have the following properties: for any $g \in L_{2}(0, T)$, the functions $g_{\xi}=\left(J_{\xi}^{-1}\right) g$ and $g_{\xi}^{*}=\left(J_{\xi}^{-1}\right)^{*} g$ are in $W_{2}^{1}(0, T)$ such that $\left.g_{\xi}\right|_{t=0}=0$ and $\left.g_{\xi}^{*}\right|_{t=T}=0$. Moreover, $J_{\xi}^{-1}$ commutes with $\partial / \partial t$, so $\int_{0}^{T}\left|g_{\xi}-g\right|^{2} d t \rightarrow 0$ and $\int_{0}^{T}\left|g_{\xi}^{*}-g\right|^{2} d t \rightarrow 0$ for $\xi \rightarrow 0$.

Now, for given $\omega(x, t)$, we introduce the function

$$
v(x, t)=\omega(x, t)-\int_{0}^{x} \frac{\omega(\xi, t)}{\ell-\xi} d \xi .
$$

Integrating by parts with respect to $\xi$, we obtain

$$
\begin{aligned}
\int_{0}^{x} v(\xi, t) d \xi & =\int_{0}^{x} \omega(\xi, t) d \xi+\int_{0}^{x} \frac{\partial}{\partial \xi}(\ell-\xi) \int_{0}^{\xi} \frac{\omega(\eta, t)}{\ell-\eta} d \eta d \xi \\
& =(\ell-x)(\omega(x, t)-v(x, t)),
\end{aligned}
$$

which implies that

$$
(\ell-x) v+J v=(\ell-x) w, \quad \int_{0}^{\ell} v(x, t) d x=0 .
$$

Then, from equality (3.2) we obtain

$$
-\int_{\Omega} \frac{\partial^{2} u}{\partial t^{2}} \overline{N v} d x d t=\int_{\Omega} A(t) \frac{\partial u}{\partial t} \bar{v} d x d t
$$

where

$$
N v=(\ell-x) v+J v, \quad A(t) u=-\frac{\partial}{\partial x}\left((\ell-x) a(x, t) \frac{\partial u}{\partial x}\right) .
$$


Replace $\partial u / \partial t$ by the smoothed function $J_{\xi}^{-1}(\partial u / \partial t)$ in (3.8) and use the relation

$$
A(t) J_{\xi}^{-1}=J_{\xi}^{-1} A(\tau)+\xi J_{\xi}^{-1} \frac{\partial A(\tau)}{\partial \tau} J_{\xi}^{-1}
$$

Then, by taking the adjoint of the operator $J_{\xi}^{-1}$, and by integrating by parts with respect to $t$ in the left-hand side, we obtain

$$
\int_{\Omega} \frac{\partial u}{\partial t} \overline{N \frac{\partial v_{\xi}^{*}}{\partial t}} d x d t=\int_{\Omega} A(t) \frac{\partial u}{\partial t} \overline{v_{\xi}^{*}} d x d t+\xi \int_{\Omega} \frac{\partial A}{\partial t}\left(\frac{\partial u}{\partial t}\right)_{\xi} \overline{v_{\xi}^{*}} d x d t
$$

The operator $A(t)$ has a continuous inverse on $L_{2}(0, \ell)$ defined by the relation

$$
A^{-1}(t) g=-\int_{0}^{x} \frac{d \xi}{a(\xi, t)(\ell-\xi)} \int_{0}^{\xi} g(\eta) d \eta+c \int_{0}^{x} \frac{d \xi}{a(\xi, t)(\ell-\xi)},
$$

where

$$
c=\frac{\int_{0}^{\ell}(d x / a(x, t)) \int_{0}^{x} g(\xi) d \xi}{\int_{0}^{\ell}(d x / a(x, t))}, \quad \int_{0}^{\ell} A^{-1}(t) g d x=0 .
$$

Hence, the function $(\partial u / \partial t)_{\xi}$ can be represented in the form

$$
\left(\frac{\partial u}{\partial t}\right)_{\xi}=J_{\xi}^{-1} A^{-1}(t) A(t) \frac{\partial u}{\partial t}
$$

Then, $(\partial A / \partial t)(\partial u / \partial t)_{\xi}=A_{\xi}(t) A(t)(\partial u / \partial t)$, where

$$
A_{\xi}(t)=\left(\frac{\partial^{2} a}{\partial x \partial t} J_{\xi}^{-1}-\frac{\partial a}{\partial t} J_{\xi}^{-1} \frac{\partial a}{\partial x} \frac{1}{a}\right) \frac{1}{a}\left(\int_{0}^{x} g(\eta, t) d \eta-c\right)+\frac{\partial a}{\partial t} J_{\xi}^{-1} \frac{1}{a} g
$$

where the constant $c$ is given by (3.13).

Consequently, equation (3.11) becomes

$$
\int_{\Omega} \frac{\partial u}{\partial t} \overline{N \frac{\partial v_{\xi}^{*}}{\partial t}} d x d t=\int_{\Omega} A(t) \frac{\partial u}{\partial t}\left(v_{\xi}^{*}+\xi A_{\xi}^{*} v_{\xi}^{*}\right) d x d t
$$

in which the conjugate operator $A_{\xi}^{*}(t)$ of $A_{\xi}(t)$ is defined by

$$
A_{\xi}^{*} v_{\xi}^{*}=\frac{1}{a}\left(J_{\xi}^{-1}\right)^{*} \frac{\partial a}{\partial \tau} v_{\xi}^{*}+\left(B v_{\xi}^{*}\right)(x)-\left(B v_{\xi}^{*}\right)(0) \frac{\int_{x}^{\ell}(d \xi / a(\xi, t))}{\int_{0}^{\ell}(d \xi / a(\xi, t))},
$$

where

$$
\left(B v_{\xi}^{*}\right)(x)=\int_{x}^{\ell} \frac{1}{a(\xi, t)}\left[\left(J_{\xi}^{-1}\right)^{*} \frac{\partial^{2} a}{\partial \xi \partial \tau}-\frac{1}{a(\xi, t)} \frac{\partial a}{\partial \xi}\left(J_{\xi}^{-1}\right)^{*} \frac{\partial a}{\partial \tau}\right] v_{\xi}^{*}(\xi, \tau) d \xi .
$$


The left-hand side of (3.16) is a continuous linear functional of $\partial u / \partial t$. Hence, the function $h_{\xi}=v_{\xi}^{*}+\xi A_{\xi}^{*} v_{\xi}^{*}$ has the derivatives $(\ell-x)\left(\partial h_{\xi} / \partial x\right) \in L_{2}(\Omega),(\partial / \partial x)((\ell-$ $\left.x)\left(\partial h_{\xi} / \partial x\right)\right) \in L_{2}(\Omega)$, and the following conditions are satisfied

$$
\left.h_{\xi}\right|_{x=0}=0,\left.\quad h_{\xi}\right|_{x=\ell}=0,\left.\quad(\ell-x) \frac{\partial h_{\xi}}{\partial x}\right|_{x=\ell}=0
$$

From (3.17) we have

$$
\begin{gathered}
(\ell-x) \frac{\partial h_{\xi}}{\partial x}=\left(I+\xi \frac{1}{a}\left(J_{\xi}^{-1}\right)^{*} \frac{\partial a}{\partial \tau}\right) \frac{\partial v_{\xi}^{*}}{\partial x}, \\
\frac{\partial}{\partial x}\left((\ell-x) \frac{\partial h_{\xi}}{\partial x}\right)=\left(I+\xi \frac{1}{a}\left(J_{\xi}^{-1}\right)^{*} \frac{\partial a}{\partial \tau}\right) \frac{\partial}{\partial x}\left((\ell-x) \frac{\partial v_{\xi}^{*}}{\partial x}\right) \\
+\xi\left[-\frac{(\partial a / \partial x)\left(J_{\xi}^{-1}\right)^{*}(\partial a / \partial \tau)}{a^{2}}+\frac{1}{a}\left(J_{\xi}^{-1}\right)^{*} \frac{\partial^{2} a}{\partial x \partial \tau}\right](\ell-x) \frac{\partial v_{\xi}^{*}}{\partial x}, \\
{\left[\left(I+\xi \frac{1}{a}\left(J_{\xi}^{-1}\right)^{*} \frac{\partial a}{\partial \tau}\right) v_{\xi}^{*}\right]_{x=0}=0,} \\
{\left[\left(I+\xi \frac{1}{a}\left(J_{\xi}^{-1}\right)^{*} \frac{\partial a}{\partial \tau}\right)(\ell-x) \frac{\partial v_{\xi}^{*}}{\partial x}\right]_{x=\ell}=0 .}
\end{gathered}
$$

Since $\left\|\xi(1 / a)\left(J_{\xi}^{-1}\right)^{*}(\partial a / \partial \tau)\right\|_{L_{2}(\Omega)}<1$ for sufficiently small $\xi$, the operator $I+$ $\xi(1 / a)\left(J_{\xi}^{-1}\right) *(\partial a / \partial \tau)$ has a continuous inverse on $L_{2}(\Omega)$. In addition, the derivative of the above operator with respect to $x$ is a bounded operator in $L_{2}(\Omega)$. Therefore, from (3.20) and (3.21), the function $v_{\xi}^{*}$ has derivatives $(\ell-x)\left(\partial v_{\xi}^{*} / \partial x\right) \in L_{2}(\Omega)$ and $(\partial / \partial x)\left((\ell-x)\left(\partial v_{\xi}^{*} / \partial x\right)\right) \in L_{2}(\Omega)$.

In a similar way, we show that for each fixed $x \in[0, \ell]$ and sufficiently small $\xi$, the operator $I+\xi(1 / a)\left(J_{\xi}^{-1}\right) *(\partial a / \partial \tau)$ has a continuous inverse on $L_{2}(0, T)$; hence, (3.22), and (3.23), and (3.24) imply that

$$
\left.v_{\xi}^{*}\right|_{x=0}=0,\left.\quad v_{\xi}^{*}\right|_{x=\ell}=0,\left.\quad(\ell-x) \frac{\partial v_{\xi}^{*}}{\partial x}\right|_{x=\ell}=0 .
$$

So, for $\xi$ sufficiently small, the function $v_{\xi}^{*}$ has the same properties as $h_{\xi}$. In addition, $v_{\xi}^{*}$ satisfies the integral condition in (3.7).

Putting $u=\int_{0}^{t} \int_{0}^{\tau} \exp (c \eta) v_{\xi}^{*}(\eta, \tau) d \eta d \tau$ in (3.8), where the constant $c$ satisfies $c a_{0}-$ $a_{3}-a_{3}^{2} / a_{0} \geq 0$, and using (3.4), we obtain

$$
\int_{\Omega} \exp (c t) v_{\xi}^{*} \overline{N v} d x d t=-\int_{\Omega} A(t) \frac{\partial u}{\partial t} \exp (-c t) \frac{\partial^{2} \bar{u}}{\partial t^{2}} d x d t+\xi \int_{\Omega} A(t) \frac{\partial u}{\partial t} \frac{\partial \overline{v_{\xi}^{*}}}{\partial t} d x d t
$$


Integrating by parts each term in the left-hand side of (3.26) and taking the real parts yield

$$
\begin{aligned}
& \operatorname{Re} \int_{\Omega} A(t) \frac{\partial u}{\partial t} \exp (-c t) \frac{\partial^{2} \bar{u}}{\partial t^{2}} d x d t \\
& \geq \frac{c}{2} \int_{\Omega}(\ell-x) a(x, t) \exp (-c t)\left|\frac{\partial^{2} u}{\partial x \partial t}\right|^{2} d x d t \\
& \quad-\frac{1}{2} \int_{\Omega}(\ell-x) \frac{\partial a}{\partial t} \exp (-c t)\left|\frac{\partial^{2} u}{\partial x \partial t}\right|^{2} d x d t \\
& \operatorname{Re}\left(-\xi \int_{\Omega} A(t) \frac{\partial u}{\partial t} \frac{\partial \overline{v_{\xi}^{*}}}{\partial t} d x d t\right) \geq \frac{-\xi a_{3}^{2}}{2 a_{0}} \int_{\Omega}(\ell-x) \exp (-c t)\left|\frac{\partial^{2} u}{\partial x \partial t}\right|^{2} d x d t .
\end{aligned}
$$

Now, using (3.27) in (3.26) with the choice of $c$ indicated above we have

$$
2 \operatorname{Re} \int_{\Omega} \exp (c t) v_{\xi}^{*} \overline{N v} d x d t \leq 0 .
$$

Then, for $\xi \rightarrow 0$ we obtain $2 \operatorname{Re} \int_{\Omega} \exp (c t) v \overline{N v} d x d t \leq 0$, that is,

$$
2 \operatorname{Re} \int_{\Omega} \exp (c t)(\ell-x)|v|^{2} d x d t+2 \operatorname{Re} \int_{\Omega} \exp (c t) v J \bar{v} d x d t \leq 0 .
$$

Since $\operatorname{Re} \int_{\Omega} \exp (c t) v J \bar{v} d x d t=0$, we conclude that $v=0$; hence, $\omega=0$, which ends the proof of the lemma.

THEOREM 3.2. The range $R(L)$ of $L$ coincides with $F$.

Proof. Since $F$ is a Hilbert space, we have $R(L)=F$ if and only if the relation

$\int_{\Omega}(\ell-x)^{2} \mathscr{L} u \bar{f} d x d t+\int_{0}^{\ell}\left[(\ell-x)^{2}\left(\frac{d l_{1} u}{d x} \frac{d \bar{\varphi}}{d x}+\frac{d l_{2} u}{d x} \frac{d \bar{\psi}}{d x}\right)\right] d x+\int_{0}^{\ell}\left(l_{1} u \bar{\varphi}+l_{2} u \bar{\psi}\right) d x=0$,

for arbitrary $u \in E$ and $(f, \varphi, \psi) \in F$, implies that $f=0, \varphi=0$ and $\psi=0$. Putting $u \in D_{0}(L)$ in (3.30), we conclude from Lemma 3.1 that $(\ell-x) f=0$. Hence,

$$
\int_{0}^{\ell}\left[(\ell-x)^{2}\left(\frac{d l_{1} u}{d x} \frac{d \bar{\varphi}}{d x}+\frac{d l_{2} u}{d x} \frac{d \bar{\psi}}{d x}\right)+l_{1} u \bar{\varphi}+l_{2} u \bar{\psi}\right] d x=0 \quad \forall u \in D(L) .
$$

Setting

$$
D_{0 k}(L)=\left\{u \in D(L):\left.u^{(k)}\right|_{t=0}=0, k=0,1\right\},
$$

and taking $u \in D_{01}(L)$ in (3.31) yield

$$
\int_{0}^{\ell}\left[(\ell-x)^{2} \frac{d l_{1} u}{d x} \frac{d \bar{\varphi}}{d x}+l_{1} u \bar{\varphi}\right] d x=0 .
$$

The range of the trace operator $l_{1}$ is everywhere dense in Hilbert space with the norm $\left[\int_{0}^{\ell}\left((\ell-x)^{2}|d \varphi / d x|^{2}+|\varphi|^{2}\right) d x\right]^{1 / 2}$; hence, $\varphi=0$. Likewise, for $u \in D_{00}(L)$, we get $\psi=0$. 


\section{REFERENCES}

[1] G. W. Batten, Jr., Second-order correct boundary conditions for the numerical solution of the mixed boundary problem for parabolic equations, Math. Comp. 17 (1963), 405-413. MR 27\#6399. Zbl 133.38601.

[2] A. Bouziani and N.-E. Benouar, Mixed problem with integral conditions for a third order parabolic equation, Kobe J. Math. 15 (1998), no. 1, 47-58. MR 99j:35087. Zbl 921.35068.

[3] J. R. Cannon, The solution of the heat equation subject to the specification of energy, Quart. Appl. Math. 21 (1963), 155-160. MR 28\#3650.

[4] _ The One-dimensional Heat Equation, Encyclopedia of Mathematics and its Applications, vol. 23, Addison-Wesley Publishing, Massachusetts, 1984. MR 86b:35073. Zbl 567.35001.

[5] J. R. Cannon, S. Pérez Esteva, and J. van der Hoek, A Galerkin procedure for the diffusion equation subject to the specification of mass, SIAM J. Numer. Anal. 24 (1987), no. 3, 499-515. MR 88e:65132. Zbl 677.65108.

[6] Y. S. Choi and K.-Y. Chan, A parabolic equation with nonlocal boundary conditions arising from electrochemistry, Nonlinear Anal. 18 (1992), no. 4, 317-331. MR 93b:35057. Zbl 757.35031.

[7] R. E. Ewing and T. Lin, A class of parameter estimation techniques for fluid flow in porous media, Adv. in Water Res. 14 (1991), no. 2, 89-97. MR 92b:65080.

[8] N. I. Ionkin, Loesung eines Randwertproblems der Waermeleitungstheorie mit einer nichtklassischen Randwertbedingung [The solution of a certain boundary value problem of the theory of heat conduction with a nonclassical boundary condition], Differencial'nye Uravnenija 13 (1977), no. 2, 294-304 (Russian). MR 58\#29240a. Zbl 349.35040.

[9] L. I. Kamynin, A boundary value problem in the theory of heat conduction with a nonclassical boundary condition, U.S.S.R. Comput. Math. and Math. Phys. 4 (1964), no. 6, 33-59. Zbl 206.39801.

[10] A. V. Kartynnik, Three-point boundary-value problem with an integral space-variable condition for a second-order parabolic equation, Differential Equations 26 (1990), no. 9, 1160-1166. Zbl 729.35053.

[11] P. Shi, Weak solution to an evolution problem with a nonlocal constraint, SIAM J. Math. Anal. 24 (1993), no. 1, 46-58. MR 93m:35090. Zbl 810.35033.

[12] P. Shi and M. Shillor, On design of contact patterns in one-dimensional thermoelasticity, Theoretical Aspects of Industrial Design (Wright-Patterson Air Force Base, OH, 1990), SIAM, Pennsylvania, 1992, pp. 76-82. MR 93e:73005.

[13] N. I. Yurchuk, Mixed problem with an integral condition for certain parabolic equations, Differential Equations 22 (1986), 1457-1463. Zbl 654.35041.

M. Denche: Institut De MathÉmatiques, Université Mentouri Constantine, ConstanTINE 25000, ALGERIA

E-mail address: m_denche@hotmai 1 .com

A. L. MARHoune: Institut De MathÉmatiQues, Université Mentouri Constantine, ConSTANTINE 25000, ALGERIA 


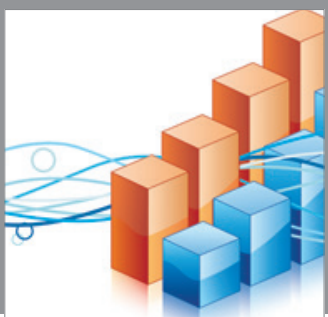

Advances in

Operations Research

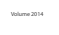

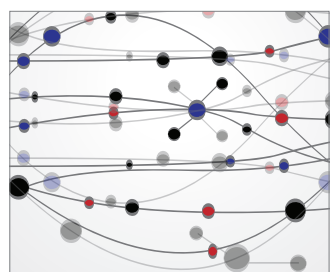

\section{The Scientific} World Journal
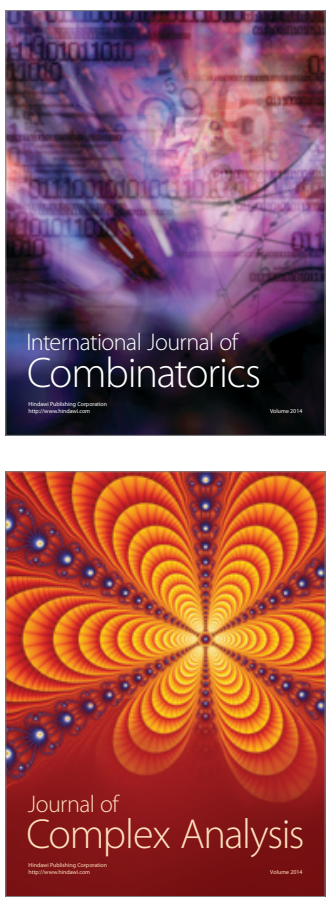

International Journal of

Mathematics and

Mathematical

Sciences
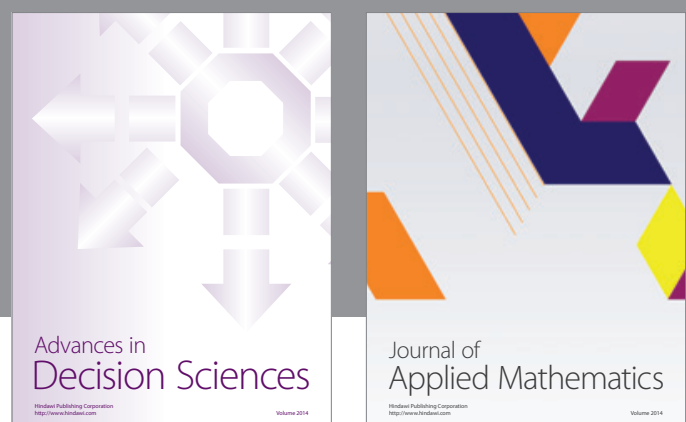

Journal of

Applied Mathematics
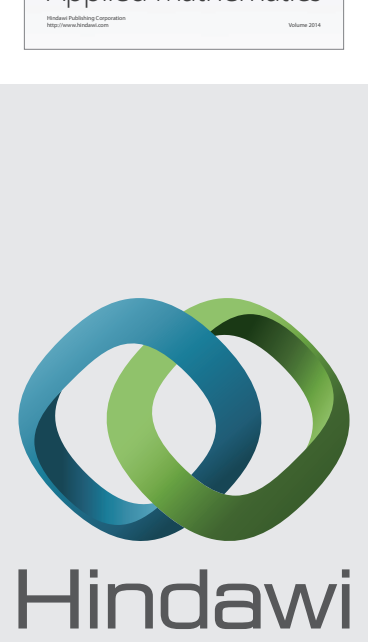

Submit your manuscripts at http://www.hindawi.com
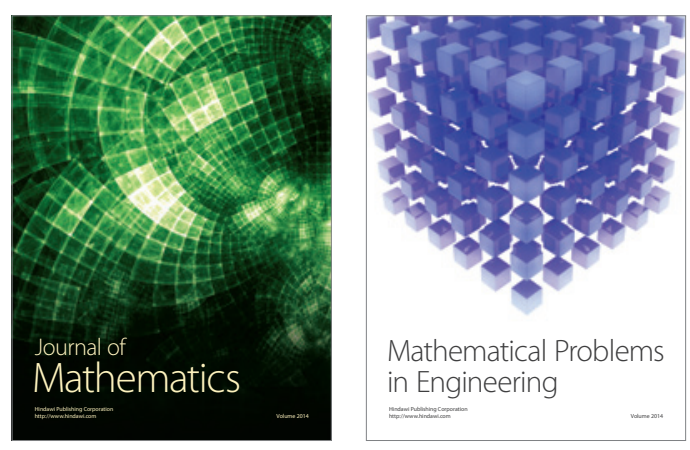

Mathematical Problems in Engineering
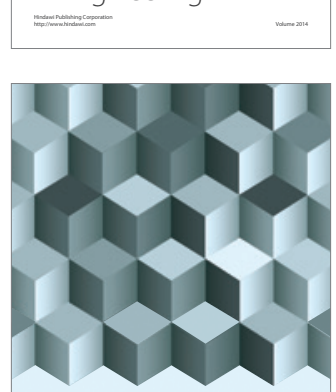

Journal of

Function Spaces
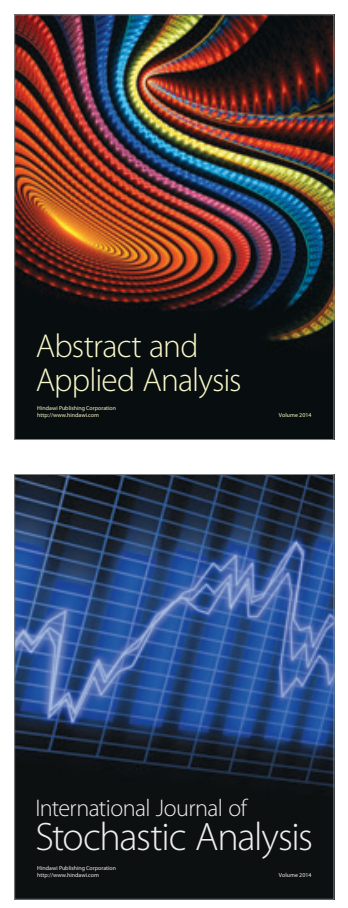

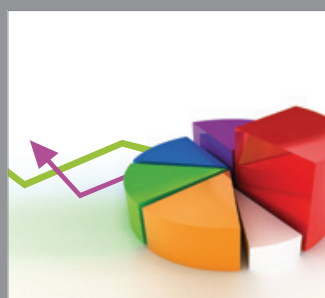

ournal of

Probability and Statistics

Promensencen
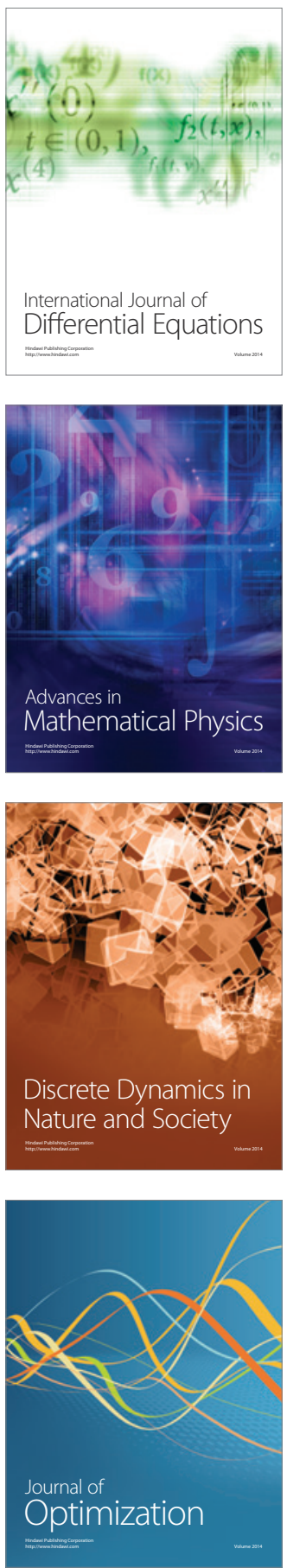